\title{
Cytomorphologic features as predictors of aggressiveness in patients with pT1 papillary thyroid carcinoma: a retrospective study of associations with clinicopathological parameters in 226 fine-needle aspirates
}

\author{
Yanli Zhu ${ }^{1 \#}$, Wenhao Ren ${ }^{1 \#}$, Yuntao Song ${ }^{2}$, Zhihui Fan ${ }^{3}$, Qian Wang ${ }^{1}$, Haizhu Jin ${ }^{1}$, Yiyi Guo ${ }^{1}$, Yanhua Bai ${ }^{1}$ \\ ${ }^{1}$ Key laboratory of Carcinogenesis and Translational Research (Ministry of Education), Department of Pathology, Peking University Cancer Hospital \\ and Institute, Beijing, China; ${ }^{2}$ Key laboratory of Carcinogenesis and Translational Research (Ministry of Education), Department of Head and Neck \\ Surgery, Peking University Cancer Hospital and Institute, Beijing, China; ${ }^{3}$ Key laboratory of Carcinogenesis and Translational Research (Ministry of \\ Education), Department of Ultrasound, Peking University Cancer Hospital and Institute, Beijing, China \\ Contributions: (I) Conception and design: Y Zhu, W Ren, Y Bai; (II) Administrative support: Y Bai; (III) Provision of study materials or patients: Y \\ Song, Z Fan, Q Wang, H Jin, Y Guo; (IV) Collection and assembly of data: Y Zhu, W Ren; (V) Data analysis and interpretation: Y Zhu, W Ren, Y \\ Bai; (VI) Manuscript writing: All authors; (VII) Final approval of manuscript: All authors. \\ \#These authors contributed equally to this work. \\ Correspondence to: Yanhua Bai, MD. Key Laboratory of Carcinogenesis and Translational Research (Ministry of Education), Department of Pathology, \\ Peking University Cancer Hospital and Institute, Beijing 100142, China. Email: 15011531378@139.com.
}

\begin{abstract}
Background Distinguishing aggressive pT1 papillary thyroid carcinomas (PTCs) from indolent PTCs before or during surgery is important. To the best of our knowledge, few reports in the literature have examined the value of the cytomorphologic features of PTC as predictors of aggressiveness.

Methods: This retrospective study included 226 pT1 PTC patients who underwent preoperative fineneedle aspiration cytology (FNAC) and surgery at Peking University Cancer Hospital between January 2018 and December 2019. Data on the clinical characteristics and pathological results were obtained from the electronic medical record database. All FNAC smears were blindly reviewed by two independent cytopathologists, and the associations between nine cytomorphologic features (lymphocytes, multinucleated giant cells, cellularity, cellular adhesiveness, nuclear size, nuclear pleomorphism, nuclear membrane regularity, intranuclear pseudoinclusions and the amount of cytoplasm) and clinicopathological parameters were statistically analyzed.
\end{abstract}

Results: Univariate analysis showed that cellularity, intranuclear pseudoinclusions, cellular adhesiveness, nuclear size, and nuclear pleomorphism were strong predictors of some clinicopathological parameters such as extracapsular invasion (ECI) and lymph node metastasis (LNM). Multivariate analysis confirmed that cellular adhesiveness was a strong independent predictor of ECI $(\mathrm{P}=0.001)$ and $\mathrm{LNM}(\mathrm{P}<0.001)$, and the amount of cytoplasm can also predict LNM $(\mathrm{P}=0.024)$.

Conclusions: Cytomorphologic features including cellular adhesiveness and the amount of cytoplasm in preoperative FNAC smears could be a valuable tool for predicting ECI or LNM and may be predictors of aggressiveness in patients with pT1 PTC.

Keywords: Thyroid; papillary thyroid carcinoma (PTC); cytomorphology; aggressiveness; fine needle aspiration

Submitted Jul 20, 2020. Accepted for publication Nov 04, 2020.

doi: 10.21037 /gs-20-618

View this article at: http://dx.doi.org/10.21037/gs-20-618 


\section{Introduction}

Papillary thyroid carcinoma (PTC) is the most common thyroid malignancy, and most PTCs, especially those with a small diameter, are characterized by indolent clinical behavior and excellent survival. However, a small proportion of PTCs manifest highly aggressive clinical behaviors, such as high rates of extracapsular invasion (ECI), multifocality, nodal and distant metastasis, and recurrence (1-9). Some aggressive PTCs are recognized by the World Health Organization (WHO) as biologically aggressive variants (10), including tall cell, columnar cell, solid, hobnail, diffuse sclerosing, and diffuse follicular variants, while some nonaggressive subtypes (classic or follicular subtype) can also manifest highly aggressive clinical behaviors.

It is very important to distinguish aggressive PTCs from indolent PTCs before or during surgery, which will allow formulation of an appropriate management plan involving more aggressive surgery, adjuvant therapy and closer surveillance. However, intraoperative frozen section examination of invasive clinical manifestations is still unsatisfactory, as it cannot provide an accurate subtype and has a high false negative rate for lymph node metastasis (LNM) (11,12).

Fine-needle aspiration cytology (FNAC) has been widely used as the most effective preoperative evaluation tool to distinguish benign from malignant lesions $(13,14)$. However, diagnosis of aggressive PTC subtypes by preoperative FNAC may not be possible or reliable even for a skilled cytopathologist. The main reasons are as follows: (I) many PTCs are heterogeneous with more than one cell type and/ or growth pattern, and the predominant pattern may not have been sampled by FNAC; (II) the cytomorphologic features among different PTC subtypes have significant overlap (15-18). For example, some of the described hobnail PTC features are also shared by the tall cell variant (16); and (III) the rarity of these aggressive variants makes it very difficult for cytopathologists to become familiar with their morphologic features, and interobserver variability exists in their interpretation (19-22). Therefore, several authors have stressed the role of molecular tests, such as BRAF analysis, performed on cytologic material from FNA to determine the extent of surgery $(23,24)$; however, not all samples have sufficient materials for molecular detection, especially small focal PTCs.

Although precise subtyping may not be possible or reliable on a cytological basis alone, awareness of the aggressive cytomorphologic features may provide indications for aggressive behavior, which will aid in the clinical management of PTC, especially stage T1 PTCs (13). To the best of our knowledge, few reports in the literature have described the value of the cytomorphologic features of PTC as predictors of aggressiveness (25). Therefore, we analyzed and evaluated nine cytological morphological features by reviewing the FNA smears of 226 pT1 PTC patients, and the correlations between these features and the clinicopathological parameters were evaluated to determine the morphological features indicating aggressiveness. This article is presented in accordance with the STROBE reporting checklist (available at http://dx.doi.org/10.21037/gs-20-618).

\section{Methods}

\section{Materials}

The inclusion criteria were as follows: (I) the patient underwent preoperative FNAC and surgery at Peking University Cancer Hospital between January 2018 and December 2019; (II) the histological pathology of the patient indicated pT1 PTC classified by AJCC 8th edition staging system, with tumor $2 \mathrm{~cm}$ or less in the greatest dimension and minimal microscopic invasion. The exclusion criteria included FNAC smears comprising only a liquidbased smear. According to these criteria, 226 patients were included in the study. Data on the clinical characteristics and pathological results were obtained from the electronic medical record database of our institution. FNAs were collected by surgeons or sonographers via ultrasound guidance, and ultrasound evaluation was carried out before puncture. In the case of multiple suspicious malignant nodules, if the suspicious malignant nodules were distributed in different gland lobes, the most suspicious nodule in each lobe was selected for puncture separately. If multiple suspicious malignant nodules were located in the same gland and the degree of malignancy was similar, the operator selected the nodule with the largest diameter, the nodule with ECI or the nodule with invasion into the surrounding structures such as strap muscles, trachea, larynx, vasculature, esophagus, and/or recurrent laryngeal nerve.

The aspirates were prepared as conventional smears stained with hematoxylin-eosin and/or as liquid-based cytology samples stained with Papanicolaou stain. In our study, 141 of the 226 samples included only conventional smears, and the remaining 85 samples included conventional smears and liquid-based smears. The cytologic diagnosis of the FNAC specimen was performed according to the 


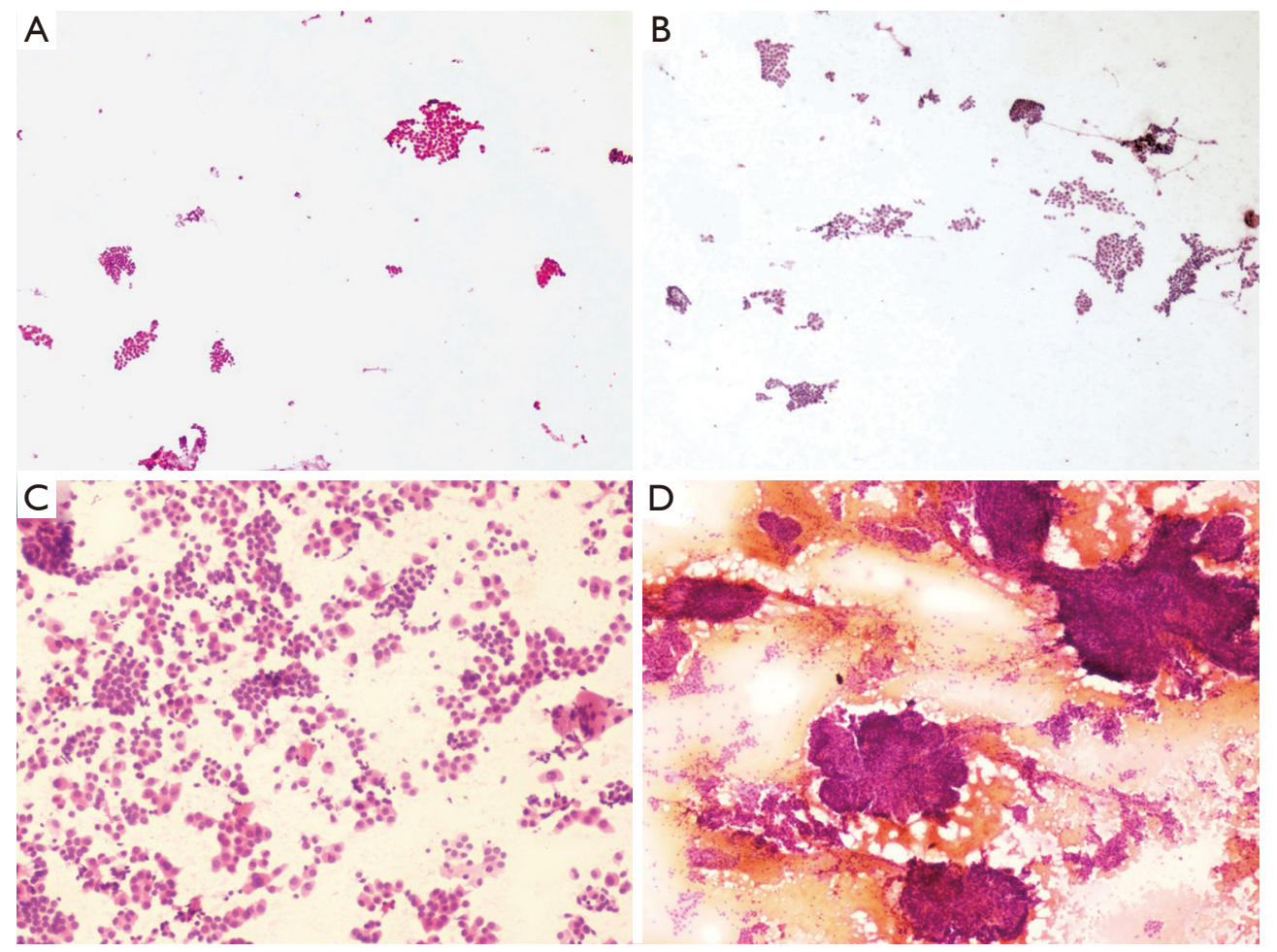

Figure 1 Microscopic overview of the tumor cells showing different cellular adhesiveness (HE, magnification 40×). (A) The tumor cells arranged predominantly small sheet-like; it was given a score of 0 . (B) The tumor architectural features consisted of small sheet-like arrangement, with few discohesive single cells and was scored 1. (C) The cell adhesion was poor and showed predominantly isolated pattern; it was given a score of 2. (D) The tumor cells gave a manifestation of overproliferation and the smear was almost entirely composed of solid clusters, it was also given a score of 2 .

Bethesda system for reporting thyroid cytopathology (TBSRTC), and the following diagnostic terminology was used according to the 6 diagnostic categories (DCs) recommended by TBSRTC: nondiagnostic or unsatisfactory (ND/UNS; DC I); benign (B; DC II); atypia of undetermined significance or follicular lesion of undetermined significance (AUS/FLUS; DC III); suspicious for follicular neoplasm or follicular neoplasm (SFN/FN; DC IV); suspicious for malignancy (SM; DC V); and malignant (M; DC VI). Only DC V and DC VI specimens were included in our series. The study was conducted in accordance with the Declaration of Helsinki (as revised in 2013). The study was approved by the Ethics Committee of the Peking University Cancer Hospital (approval ID: No. 2018KT101), and informed consent was obtained from all patients.

\section{Cytomorphologic features}

For each case, all FNAC smears were reviewed blindly by two independent cytopathologists (Yanli Zhu and Wenhao Ren). The cytomorphologic features examined were as follows: (I) background components (lymphocytes and multinucleated giant cells); (II) architectural features (cellularity and cellular adhesiveness); and (III) nuclei and cytoplasm features (nuclear size, nuclear pleomorphism, nuclear membrane regularity, presence of intranuclear pseudoinclusions and the amount of cytoplasm). Conventional smears were used to examine the architectural features, background components and several nuclei and cytoplasm features (nuclear size, presence of intranuclear pseudoinclusions and the amount of cytoplasm), and liquid-based smears were used to evaluate the nuclear pleomorphism and nuclear membrane regularity.

To obtain a semiquantitative evaluation, a score of 0 to 2 was assigned to each feature. For example, for cellular adhesiveness (Figure 1), the degree of aggregation between cells was divided into 3 levels as follows: predominantly a small sheet-like arrangement (score 0), few discohesive single 


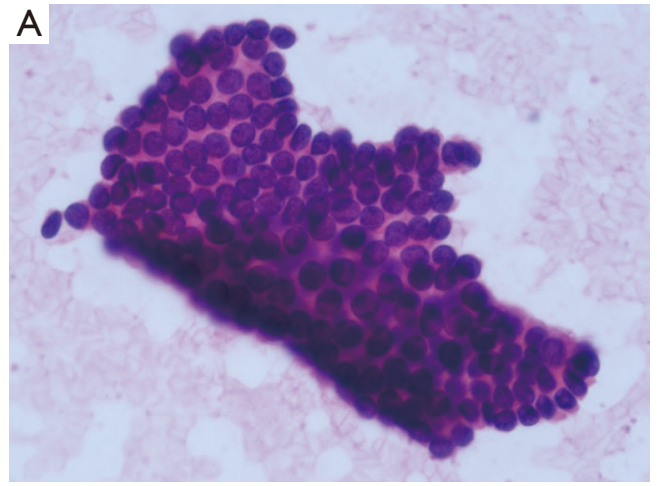

B
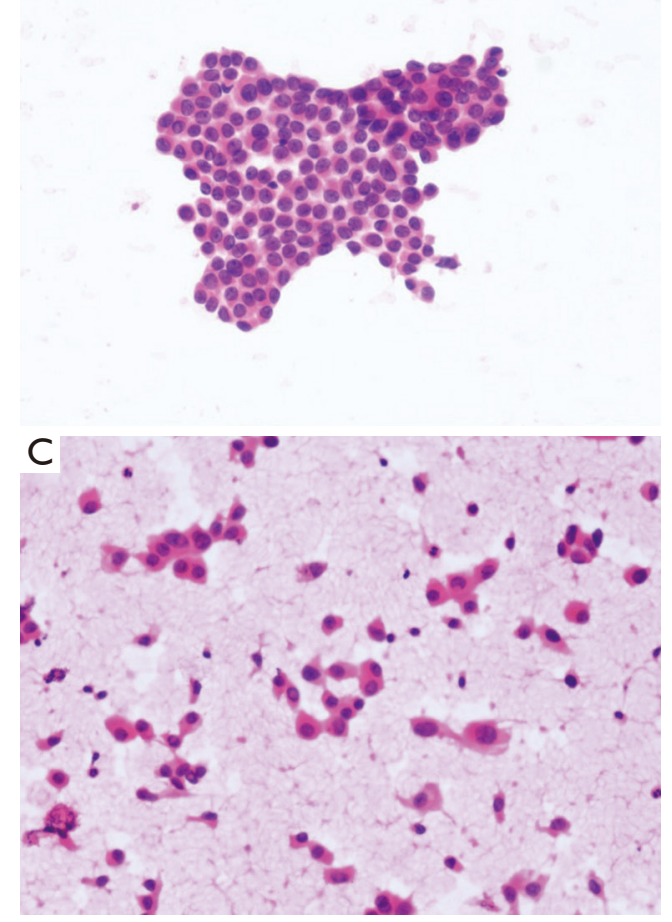

Figure 2 The tumor cells differed in the amount of cytoplasm (HE, magnification 200x). (A) The nuclear to cytoplasmic ratios of almost all tumor cells were high, with scant cytoplasm; it was given a score of 0 . (B) The tumor cells had a medium amount of cytoplasm, between scant and abundant, and was scored 1. (C) When the radius of the cell was more than 2 times the radius of the nuclear, the amount of cytoplasm was defined as abundant, and the smear was almost entirely composed of these cells; it was also given a score of 2 .

cells (score 1) and predominantly an isolated pattern or solid clusters (score 2); for amount of cytoplasm (Figure 2), the degree was as follows: scant (score0), medium (score1) and abundant (score 2). The parameters used to evaluate the cytomorphologic features of PTC in FNAC specimens are shown in Table 1 and the accessory notes. When the final scores of the two observers were not identical for a given case, a consensus was reached after discussion.

\section{Clinicopathological parameters}

Surgical pathology is the gold standard, and the clinicopathological parameters of tumor size, tumor focality, ECI and LNM in our study were based on the surgical pathology. ECI is defined as minimal microscopic invasion, without invasion into the surrounding structures such as strap muscles, trachea, larynx, vasculature, esophagus, and/ or recurrent laryngeal nerve.

\section{Statistical analysis}

Univariate analysis using the $\chi^{2}$ test and multivariable analysis using logistic regression were performed to determine the important cytomorphologic features and clinicopathological parameters in patients with PTC. The level of statistical significance was set at $\mathrm{P}<0.05$. Data were analyzed with the Statistical Package for the Social Sciences, version 19.0 (IBM Corp., Armonk, NY, USA).

\section{Results}

The study included 119 patients $(52.7 \%)$ younger than 45 years and 107 patients $(47.3 \%)$ older than 45 years. There were 171 women and 55 men (female-to-male ratio, 3.1:1). Tumors with a maximum diameter $\leq 1 \mathrm{~cm}$ were found in $163(72.1 \%)$ patients, and larger tumors were present in $63(27.9 \%)$ patients. Multifocal disease was found in $78(34.5 \%)$ patients, and ECI was found in $109(48.2 \%)$ patients. LNMs were found in $76(33.6 \%)$ patients, and none of the patients had gross extrathyroidal extension or distant metastases.

The cytological diagnosis indicated 18 (8.0\%) DC V cases and 208 (92.0\%) DC VI cases.

A univariate analysis of cytomorphologic features and clinicopathological parameters, including age, sex, tumor size, tumor focality, ECI and LNM, was carried out (Table 2). The results indicated that cellularity, intranuclear pseudoinclusions, cellular adhesiveness, nuclear size, and nuclear pleomorphism were strong predictors of some clinicopathological parameters such as ECI and LNM, whereas lymphocytes, nuclear membrane regularity, and multinucleated giant cells were not predictors. 
Table 1 Parameters used in the evaluation of cytomorphologic features of PTC in FNAC specimens

\begin{tabular}{|c|c|c|c|}
\hline Parameter & Score 0 & Score 1 & Score 2 \\
\hline Lymphocytes & Absent & Occasional & Frequent \\
\hline Multinucleated giant cells & Absent & Occasional & Frequent \\
\hline \multicolumn{4}{|l|}{ Architectural features } \\
\hline Cellular adhesiveness & $\begin{array}{l}\text { Predominantly a small sheet-like } \\
\text { arrangement }\end{array}$ & Few discohesive single cells & $\begin{array}{l}\text { Predominantly an isolated } \\
\text { pattern or solid clusters }\end{array}$ \\
\hline \multicolumn{4}{|l|}{ Nuclei and cytoplasm } \\
\hline Nuclear size & Absence of large nuclei & Few large nuclei & Predominantly large nuclei \\
\hline Intranuclear pseudoinclusions & Absent & Occasional & Conspicuous \\
\hline Amount of cytoplasm & Scant & Medium & Abundant \\
\hline
\end{tabular}

Large nuclei refer to nuclei at least three times the size of a granulocyte. Nuclear pleomorphism was evaluated in terms of variations in shape and size among the nuclei in the same smear. When the radius of the cell was more than 2 times the radius of the nucleus, the amount of cytoplasm was considered abundant. PTC, papillary thyroid carcinoma; FNAC, fine-needle aspiration cytology.

Table $2 \mathrm{P}$ value of univariate analysis of the cytomorphologic features and clinicopathological parameters in patients with papillary thyroid carcinoma

\begin{tabular}{|c|c|c|c|c|c|c|c|c|c|c|c|c|}
\hline & \multicolumn{2}{|c|}{ Age $(n, \%)$} & \multicolumn{2}{|c|}{$\operatorname{Sex}(n, \%)$} & \multicolumn{2}{|c|}{ Tumor size (n, \%) } & \multicolumn{2}{|c|}{ Focality (n, \%) } & \multicolumn{2}{|c|}{$\begin{array}{l}\text { Extracapsular } \\
\text { invasion (n, \%) }\end{array}$} & \multicolumn{2}{|c|}{$\begin{array}{c}\text { Lymph node } \\
\text { metastases (n, \%) }\end{array}$} \\
\hline & $<45$ & $\geq 45$ & Female & Male & $<1$ & $\geq 1$ & Unifocal & Multifocal & - & + & - & + \\
\hline Cellularity & \multicolumn{2}{|c|}{0.057} & \multicolumn{2}{|c|}{0.226} & \multicolumn{2}{|c|}{0.007} & \multicolumn{2}{|c|}{0.590} & \multicolumn{2}{|c|}{0.009} & \multicolumn{2}{|c|}{$<0.001$} \\
\hline Lymphocytes & \multicolumn{2}{|c|}{0.044} & \multicolumn{2}{|c|}{0.106} & \multicolumn{2}{|c|}{0.294} & \multicolumn{2}{|c|}{0.532} & \multicolumn{2}{|c|}{0.674} & \multicolumn{2}{|c|}{0.314} \\
\hline $\begin{array}{l}\text { Cellular } \\
\text { adhesiveness }\end{array}$ & \multicolumn{2}{|c|}{0.035} & \multicolumn{2}{|c|}{0.561} & \multicolumn{2}{|c|}{$<0.001$} & \multicolumn{2}{|c|}{0.238} & \multicolumn{2}{|c|}{$<0.001$} & \multicolumn{2}{|c|}{$<0.001$} \\
\hline $\begin{array}{l}\text { Nuclear membrane } \\
\text { regularity }\end{array}$ & & & 0.6 & 97 & & & 0.1 & 93 & 0.1 & & & \\
\hline $\begin{array}{l}\text { Multinucleated } \\
\text { giant cells }\end{array}$ & & & 0.0 & 88 & & & 0.2 & 63 & $0 . c$ & & & \\
\hline
\end{tabular}




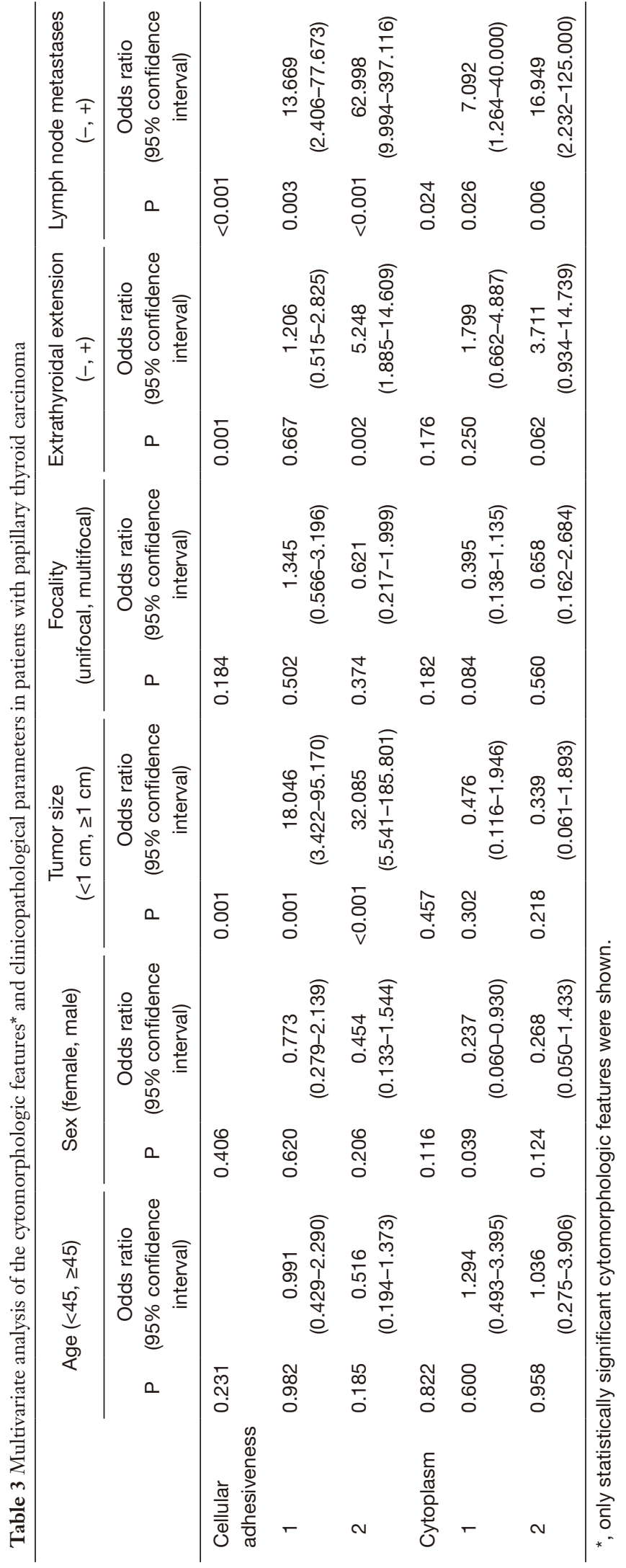

Multivariable logistic regression analysis (Table 3) further revealed that ECI was present in more patients with an adhesiveness score of 2 (predominantly the isolated pattern or solid clusters) than in those with an adhesiveness score of 0 (predominantly the small sheet-like arrangement) $(\mathrm{P}=0.002, \mathrm{OR}=5.248)$, However, no significant difference was observed between patients with an adhesiveness score of 1 (few isolated patterns or solid clusters) and those with a score of 0 . The LNM rates in patients with an adhesiveness score of 1 were significantly higher than those with a score of $0(\mathrm{OR}=13.669, \mathrm{P}<0.001)$. However, the $\mathrm{OR}$ value was $62.998(\mathrm{P}<0.001)$ when patients with an adhesiveness score of 2 were compared to those with a score of 0 . The LNM rates of patients with high cellular adhesiveness scores were significantly higher than those of patients with low cellular adhesiveness scores (OR of patients with a score of 1 compared to those with a score of $0=13.669$, OR of patients with a score of 2 compared to those with a score of $0=62.998, \mathrm{P}<0.001)$. The LNM rates of patients with high cytoplasmic scores (more cytoplasm) were significantly higher than those in patients with low cytoplasmic scores (OR of patients with a score of 1 compared to those with a score of $0=7.092$, OR of patients with a score of 2 compared to those with a score of $0=16.949, \mathrm{P}=0.024$ ).

There were no significant differences between the cytomorphologic features in the different diagnostic categories (DC V and DC VI).

\section{Discussion}

The aggressiveness of pT1 PTC is a clinical concern. Compared to pT1 PTC, the treatment course for pT2 and above is clear, which is often surgical treatment or combined radiotherapy and chemotherapy (13). For patients with pT1a PTC $(\leq 1 \mathrm{~cm})$, the decision to perform surgery or follow-up is controversial $(26,27)$, and it is necessary to conduct a comprehensive analysis based on the risk assessment and the wishes of the patient, but effective risk assessment methods are still lacking. Clinically, among patients with pT1a PTC at the same risk, some patients have LNM or ECI at an early stage, while some maintain growth in the glands for a long duration. However, although T1b patients undergo routine surgical resection, whether to perform preventive lymph node dissection for patients with a negative preoperative lymph node examination is questionable $(13,28)$. In some T1b patients with a negative preoperative lymph node examination, LNM is found in postoperative paraffin sections or LNM is found within 
a short period of time after surgery. These phenomena prompted us to consider whether it is possible to obtain evidence from cytomorphology to distinguish between aggressive and indolent cases and provide a reference for patient follow-up or surgery.

In this study, three aspects of cytomorphologic features, including background components, architectural features, and nuclei and cytoplasm features, were adopted, and we further divided these features into nine parameters (lymphocytes, multinucleated giant cells, cellularity, cellular adhesiveness, nuclear size, nuclear pleomorphism, nuclear membrane regularity, intranuclear pseudoinclusions and the amount of cytoplasm). The clinicopathological parameters included age, sex, tumor size, focality, ECI and LNM (Table 1). Although the punctured nodule may not have exhibited the same characteristics as all nodules, it was indeed the most representative since detailed ultrasound evaluation was carried out before puncture. The punctured nodule was the most malignant and aggressive nodule and its morphological characteristics were also most likely related to the clinicopathological parameters such as ECI and LNM.

The univariate analysis indicated that cellularity, intranuclear pseudoinclusions, cellular adhesiveness, nuclear size, and nuclear pleomorphism were strong predictors of some clinicopathological parameters such as ECI and LNM (Table 2). Although ECI of thyroid cancer has no prognostic significance, it is still considered an aggressive indicator in our research, a well-defined tumor capsule is a particularly favorable prognostic indicator (29).

Further multivariable logistic regression analysis (Table 3) showed that cellular adhesiveness was a strong independent predictor of ECI and LNM. ECI was found more often in patients with an adhesiveness score of 2 (predominantly the isolated pattern or solid clusters) than in those with an adhesiveness score of 0 (predominantly the small sheetlike arrangement $)(\mathrm{P}=0.002, \mathrm{OR}=5.248)$. However, no significant difference was observed between patients with an adhesiveness score of 1 ( $\mathrm{few}$ isolated patterns or solid clusters) and those with a score of 0 . The results were along with some molecular research that the growth patterns of solitary and collective maybe manifestations of invasion (30). This implied that for PTCs less than $2 \mathrm{~cm}$, when the growth pattern is predominantly isolated or exhibits solid clusters, the possibility of ECI is greatly increased.

We also found that the LNM rates in patients with high cellular adhesiveness scores were significantly higher than those in patients with low cellular adhesiveness scores (OR of patients with a score of 1 compared to those with a score of $0=13.669$, OR of patients with a score of 2 compared to those with a score of $0=62.998, \mathrm{P}<0.001)$. The LMN rates of patients with scores of 2, 1 and 0 were $62.7 \%$ (52/83), 31.3\% (21/67) and 3.9\% (3/76), respectively. With the gradual decrease in cellular adhesiveness of PTC, the possibility of LNM incrementally increased. This finding is of great practical significance in clinical work and will provide an important reference for clinical decision-making, such as whether to implement preventive lymph node dissection.

An additional cytomorphologic feature might predict LNM. In our research, the LNM rates in patients with high cytoplasm scores (more cytoplasm) were significantly higher than those in patients with low cytoplasm scores (OR of patients with a score of 1 compared to those with a score of $0=7.092$, OR of patients with a score of 2 compared to those with a score of $0=16.949, \mathrm{P}=0.024)$. The $\mathrm{LNM}$ rates of patients with scant, medium and abundant cytoplasm were $16.7 \%$ (15/90), 32.2\% (19/59) and 54.5\% (42/77), respectively. The LNM rates of PTC patients with increased cytoplasm levels were higher than those with low cytoplasm levels, which is different from our general knowledge. On the one hand, we suspect that this may be related to some aggressive subtypes of PTC with frequent LNMs, such as diffuse sclerosis and high cell and columnar cell variants, which are all characterized by abundant cytoplasm. On the other hand, for classical PTC, it reminds us that a lower nucleocytoplasmic ratio may not exclude the diagnosis of malignancy, on the contrary, the increased amount of cytoplasm may be evidence of increased invasiveness.

For some other cytomorphologic features, such as intranuclear pseudoinclusions, nuclear size, nuclear pleomorphism and nuclear membrane regularity, although we did not confirm their significance for aggressiveness in the multivariate analysis, they also showed statistically significant differences in the univariate analysis. One reason for this finding might be that the number of PTC patients in our study was too small to derive statistical conclusions. Therefore, further research is necessary to validate the value of these cytomorphologic features in predicting aggressiveness.

There were no significant differences between the cytomorphologic features of the different diagnostic categories (DC V and DC VI); however, there were few DC $\mathrm{V}$ cases, which might affect the reliability of the results. In our study, among DC V cases, 38.9\% (7/18) had an adhesiveness score of 1 (few discohesive single cells) and $22.2 \%(4 / 18)$ had an adhesiveness score of 2 (isolated pattern or solid clusters). Regarding the amount of cytoplasm, $27.8 \%$ $(5 / 18)$ of cases were classified as 'medium', and $22.2 \%(4 / 18)$ 
were classified as 'abundant'. These results suggest that some PTCs with a cytological diagnosis of DC V might show aggressive cytomorphologic features, and it is important for surgeons to be mindful of these characteristics in order to select a more comprehensive treatment plan.

Our research included an in-depth discussion of the value of cytomorphologic features in FNAC smears for predicting aggressiveness in patients with pT1 PTC, but there were some limitations of our study. First, only limited data were collected in this study, and this is a single center study, which possibly included some bias. Second, histopathological samples in this study did not undergo central review, and we did not classify the histological subtypes of PTC. It is not known whether some invasive cytological features are related to the histological subtypes. Third, molecular research is lacking. The addition of molecular data in relation to cytomorphologic features could make our results more credible, and this will be included in future studies. Finally, this was a retrospective study, and some bias may exist.

In conclusion, this study showed that FNAC was useful in the preoperative diagnosis of PTC and that the cytomorphologic features of cellular adhesiveness and the amount of cytoplasm could be a valuable tool for identifying ECI or LNM in patients with pT1 PTC. To the best of our knowledge, few reports have evaluated the value of cytomorphologic features in preoperative FNA smears to predict aggressiveness and guide clinicians in formulating more complete treatment plans. Further studies are needed to assess the efficiency of the predictive significance of cellular adhesiveness and the amount of cytoplasm regarding ECI or LNMS and to develop a cytological grading system involving preoperative FNA smears as a method to predict aggressiveness of pT1 PTC.

\section{Acknowledgments}

Funding: This work was supported by the Beijing Municipal Administration of Hospitals Incubating Program (grant number: PX2018042).

\section{Footnote}

Reporting Checklist: The authors have completed the STROBE reporting checklist. Available at http://dx.doi. org/10.21037/gs-20-618

Data Sharing Statement: Available at http://dx.doi. org/10.21037/gs-20-618

Peer Review File: Available at http://dx.doi.org/10.21037/gs20-618

Conflicts of Interest: All authors have completed the ICMJE uniform disclosure form (available at http://dx.doi. org/10.21037/gs-20-618). The authors have no conflicts of interest to declare.

Ethical Statement: The authors are accountable for all aspects of the work in ensuring that questions related to the accuracy or integrity of any part of the work are appropriately investigated and resolved. The study was conducted in accordance with the Declaration of Helsinki (as revised in 2013). The study was approved by Ethics Committee of the Peking University Cancer Hospital (approval ID: No. 2018KT101) and informed consent was obtained from all patients.

Open Access Statement: This is an Open Access article distributed in accordance with the Creative Commons Attribution-NonCommercial-NoDerivs 4.0 International License (CC BY-NC-ND 4.0), which permits the noncommercial replication and distribution of the article with the strict proviso that no changes or edits are made and the original work is properly cited (including links to both the formal publication through the relevant DOI and the license). See: https://creativecommons.org/licenses/by-nc-nd/4.0/.

\section{References}

1. Roman S, Sosa J. Aggressive variants of papillary thyroid cancer. Curr Opin Oncol 2013;25:33-8.

2. Baloch Z, LiVolsi V, Tondon R. Aggressive variants of follicular cell derived thyroid carcinoma; the so called 'Real Thyroid Carcinomas'. J Clin Pathol 2013;66:733-43.

3. Shi X, Liu R, Basolo F, et al. Differential clinicopathological risk and prognosis of major papillary thyroid cancer variants. J Clin Endocrinol Metab 2016;101:264-74.

4. Sen A, Nalwa A, Mathur S, et al. Cytomorphology of columnar cell variant of papillary carcinoma thyroid: A case report and review of the literature. Cytojournal 2014;11:27.

5. Asioli S, Erickson L, Sebo T, et al. Papillary thyroid carcinoma with prominent hobnail features: a new aggressive variant of moderately differentiated papillary carcinoma. A clinicopathologic, immunohistochemical, and molecular study of eight cases. Am J Surg Pathol 
2010;34:44-52.

6. Lee YS, Kim Y, Jeon S, et al. Cytologic, clinicopathologic, and molecular features of papillary thyroid carcinoma with prominent hobnail features: 10 case reports and systematic literature review. Int J Clin Exp Pathol 2015;8:7988-97.

7. Giorgadze TA, Scognamiglio T, Yang GC. Fine-needle aspiration cytology of the solid variant of papillary thyroid carcinoma: a study of 13 cases with clinical, histologic, and ultrasound correlations. Cancer Cytopathol 2015;123:71-81.

8. Vinciguerra GL, Noccioli N, Bartolazzi A. Diffuse follicular variant of papillary thyroid carcinoma: a case report with a revision of literature. Rare Tumors 2016;8:6536.

9. Gupta S, Ajise O, Dultz L, et al. Follicular variant of papillary thyroid cancer: encapsulated, nonencapsulated, and diffuse: distinct biologic and clinical entities. Arch Otolaryngol Head Neck Surg 2012;138:227.

10. Lloyd R, Osamura R, Kloppel G, editors. WHO classification of tumours of endocrine organs. 4th ed. Geneva, Switzerland; 2017.

11. Jozaghi Y, Richardson K, Anand S, et al. Frozen section analysis and sentinel lymph node biopsy in well differentiated thyroid cancer. J Otolaryngol Head Neck Surg 2013;42:48.

12. Ji YB, Lee D, Song C, et al. Accuracy of intraoperative determination of central node metastasis by the surgeon in papillary thyroid carcinoma. Otolaryngol Head Neck Surg 2014;150:542-7.

13. Haugen BR, Alexander EK, Bible KC, et al. 2015 American thyroid association management guidelines for adult patients with thyroid nodules and differentiated thyroid cancer. Thyroid 2016;26:1-133.

14. Zhu Y, Song Y, Xu G, et al. The Bethesda System for Reporting Thyroid Cytopathology (TBSRTC): A report of 2,781 cases in a Chinese population. Chin J Cancer Res 2020;32:140-8.

15. Gupta S, Sodhani P, Jain S, et al. Morphologic spectrum of papillary carcinoma of the thyroid: role of cytology in identifying the variants. Acta Cytol 2004;48:795-800.

16. Bellevicine C, Cozzolino I, Malapelle U, et al. Cytological and molecular features of papillary thyroid carcinoma with prominent hobnail features: a case report. Acta Cytol 2012;56:560-4.

17. Bongiovanni M, Mermod M, Canberk S, et al. Columnar cell variant of papillary thyroid carcinoma: Cytomorphological characteristics of 11 cases with histological correlation and literature review. Cancer Cytopathol 2017;125:389-97.

18. Ylagan LR, Dehner LP, Huettner PC, et al. Columnar cell variant of papillary thyroid carcinoma. Report of a case with cytologic findings. Acta Cytol 2004;48:73-7.

19. Albores-Saavedra J, Wu J. The many faces and mimics of papillary thyroid carcinoma. Endocr Pathol 2006;17:1-18.

20. Monappa V, Kudva R. Cytomorphologic diversity of papillary thyroid carcinoma. J Cytol 2017;34:183-7.

21. Pusztaszeri M, Auger M. Update on the cytologic features of papillary thyroid carcinoma variants. Diagn Cytopathol 2017;45:714-30.

22. Rossi ED, Faquin W, Pantanowitz L. Cytologic features of aggressive variants of follicular-derived thyroid carcinoma. Cancer Cytopathol 2019;127:432-46.

23. Howell GM, Nikiforova M, Carty S, et al. BRAFV600E mutation independently predicts central compartment lymph node metastasis in patients with papillary thyroid cancer. Ann Surg Oncol 2013;20:47-52.

24. Rossi ED, Martini M, Capodimonti S, et al. BRAF (V600E) mutation analysis on liquid-based cytology-processed aspiration biopsies predict bilaterality and lymph node involvement in papillary thyroid microcarcinoma. Cancer Cytopathol 2013;121:291-7.

25. Damiani S, Dina R, Eusebi V. Cytologic grading of aggressive and nonaggressive variants of papillary thyroid carcinoma. Am J Clin Pathol 1994;101:651-5.

26. Miyauchi A, Ito $\mathrm{Y}, \mathrm{Oda} \mathrm{H}$. Insights into the management of papillary microcarcinoma of the thyroid. Thyroid 2018;28:23-31.

27. Ito $\mathrm{Y}$, Miyauchi A, Oda H, et al. Revisiting low-risk thyroid papillary microcarcinomas resected without observation: was immediate surgery necessary? World J Surg 2016;40:523-8.

28. Viola D, Materazzi G, Valerio L, et al. Prophylactic central compartment lymph node dissection in papillary thyroid carcinoma: clinical implications derived from the first prospective randomized controlled single institution study. J Clin Endocrinol Metab 2015;100:1316-24.

29. Haddad R, Bischoff L, Busaidy N, et al. NCCN clinical practice guidelines in thyroid carcinoma. Vesion 2. 2019.

30. Nagai T, Ishikawa T, Minami Y, et al. Tactics of cancer invasion: solitary and collective invasion. J Biochem 2020;167:347-55.

Cite this article as: Zhu Y, Ren W, Song Y, Fan Z, Wang Q, Jin H, Guo Y, Bai Y. Cytomorphologic features as predictors of aggressiveness in patients with p T1 papillary thyroid carcinoma: a retrospective study of associations with clinicopathological parameters in 226 fine-needle aspirates. Gland Surg 2021;10(1):319-327. doi: 10.21037/gs-20-618 